\title{
Automated inference of rules with exception from past legal cases using ASP
}

\author{
Duangtida Athakravi ${ }^{1}$, Ken Satoh ${ }^{2}$, Mark Law $^{1}$, Krysia Broda $^{1}$, and \\ Alessandra Russo ${ }^{1}$ \\ 1 Imperial College London \\ \{duangtida.athakravi07, mark. law09, k. broda, a.russo\}@imperial.ac.uk \\ 2 National Institute of Informatics and Sokendai ksatoh@nii.ac.jp
}

\begin{abstract}
In legal reasoning, different assumptions are often considered when reaching a final verdict and judgement outcomes strictly depend on these assumptions. In this paper, we propose an approach for generating a declarative model of judgements from past legal cases, that expresses a legal reasoning structure in terms of principle rules and exceptions. Using a logic-based reasoning technique, we are able to identify from given past cases different underlying defaults (legal assumptions) and compute judgements that (i) cover all possible cases (including past cases) within a given set of relevant factors, and (ii) can make deterministic predictions on final verdicts for unseen cases. The extracted declarative model of judgements can then be used to make automated inference of future judgements, and generate explanations of legal decisions.
\end{abstract}

\section{Introduction}

In legal reasoning, especially in continental laws, we use written rules to make a judgement in litigation. In these written rules, principle rules and exceptions are mentioned. It is related to proof of persuasion where conditions of principle rules must be proved by the side who claims holding the conclusion of principle rules, whereas exceptions must be proved by the side who denies the conclusion. Moreover, some rules are refined by the highest court in a country (the supreme court in Japan, for example) by adding some exceptions if the current principle rules or exceptions do not capture the conclusion of the current litigation.

In this paper, we consider the problem of generating a set of case-rules from previous cases judged by the court. Suppose that the following cases are found in the conclusion of "depriving the other party of what he (or she) is entitled to expect under the contract" in commercial litigation.

Case 1: The plaintiff (the buyer) showed that the goods were delivered on time but he failed to prove that there was a damage of goods. In this case, the judge decided that the seller did not deprive the buyer of what he expects.

Case 2: The plaintiff showed that the goods were delivered on time but the goods were damaged. Then, the defendant failed to prove that the damage could be repaired. In this case, the judge decided that the buyer was deprived of what he expects.

Case 3: The plaintiff showed that the goods were delivered on time but the goods were damaged. Then, the defendant showed that the damage could be repaired and the buyer fixed an additional period of time for repair and 
the repair was completed in the additional period. In this case, the judge decided that the seller did not deprive the buyer of what he expects.

Case 4: The plaintiff showed that the goods were not delivered on time. Then, the defendant showed that the buyer fixed an additional period of time for the delivery but failed to prove that the goods were delivered in the period. In this case, the judge decided that the buyer was deprived of what he expects.

Case 5: The plaintiff showed that the goods were not delivered on time. Then, the defendant (the seller) showed that the buyer fixed an additional period of time and the goods were delivered in the period. In this case, the judge decided that the seller did not deprive the buyer of what he expects.

We would like to decide whether the following case satisfies the conclusion "depriving the other party of what he is entitled to expect under the contract":

New Case: The plaintiff showed that the goods were delivered on time but were damaged. The defendant showed that it could be repaired and showed that the buyer fixed an additional period of time for repair, but failed to prove that the repair was not completed in the additional period.

We can formalise all the factors mentioned in cases 1-5 as described below.

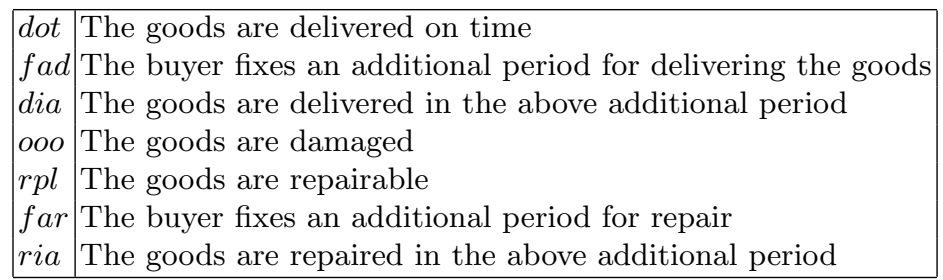

Past cases can then be expressed as pairs where the first argument denotes the factors mentioned in the case and the second argument the positive $(+d w e)$ or negative $(-d w e)$ conclusion "depriving the other party of what he is entitled to expect under the contract". We would like to predict what the conclusion would be for the new case, given the past cases:

\begin{tabular}{|l|l|l|l|}
\hline Case 1 & $\langle\{d o t\},-d w e\rangle$ & Case 4 & $\langle\{\neg d o t, f a d\},+d w e\rangle$ \\
Case 2 & $\langle\{d o t, o o o\},+d w e\rangle$ & Case 5 & $\langle\{\neg d o t, f a d, d i a\},-d w e\rangle$ \\
Case 3 & $\langle\{d o t, o o o, r p l, f a r, r i a\},-d w e\rangle$ & & \\
\hline New Case $\rangle$ & $\langle\{d o t, o o o, r p l, f a r\}, ? ?$ & \\
\hline
\end{tabular}

To make a judgement for a new case, the history of judgement revisions in past cases needs to be taken into account. In our example let us assume that if no factor is proved, the conclusion dwe will not be approved by judges (-dwe is a conclusion in an empty case - a case with an empty set of factors). Then, Case 1 has the same conclusion as the initial empty case, so the factor dot is irrelevant to the judges. Then for Case 2 the conclusion is reversed (judgement is revised). We conclude that a combination of dot and ooo does affect the conclusion and therefore we understand that the simultaneous existence of $d o t$ and ooo is an exceptional situation. Then for Case 3 the conclusion is reversed again into the original judgement. Then, we could say that the combination of rpl, far, ria is an exception to the exceptional situation in which dot and ooo exist. For Case 4, the conclusion differs from the initial empty case so the combination of $\neg$ dot and fad 
represents an exceptional situation. Lastly for Case 5 the conclusion is reversed again into the original judgement. Therefore, dia constitutes an exception for exceptional situation in which $\neg$ dot and fad exist.

Finally, for the new case, given the factors $\{$ dot,ooo, rpl,far $\}$, we should conclude $+d w e$, since $d o t$ and ooo hold in this case (and Case 2 was considered to be an exceptional situation with these factors), and it cannot be considered to be an exception for this exception since the only such exception known is the combination of rpl, far, ria, and in the new case ria does not hold.

In this paper, we aim to formalise the above reasoning and extract a set of rules that capture the past judgements and allow prediction of judgements for new cases. We view the cases and their judgements as outcomes of reasoning using an argumentation model [3], where the factors are arguments presented to the judge and the judgement is the conclusion after applying the attacks between the factors. Hence, attacks can be inferred from past cases that share some common factors but have opposing judgements, and consequently arguments are inferred from the factors that are uncommon between the two cases. Then, the purpose of relevant attacks is to identify only the necessary factors that affect the judgement of a new case. Consider the above cases. Case 1 does not contain any relevant information for deciding the outcome of future cases. Similarly, suppose Case 6 exists where the buyer was deprived of what he expects as the goods are delivered on time, but are damaged and repairable $\langle\{d o t, o o o, r p l\},+d w e\rangle$. This Case 6 will also be deemed irrelevant, due to the existence of Case 2 which tells us that $\{d o t, o o o\}$ are already sufficient for overturning the judgement. Thus, by extracting information about the relevant attacks and their arguments we can define rules reflecting the reasoning applied by the judge. A meta-level representation of these rules is generated using the ASP solver Clingo [4].

The paper is structured as follows. In Section 2 we provide the formal definition of our computational model, introduce the notions of relevant attack and prediction of judgement. In Section 3 we show how the approach is implemented in Answer Set Programming (ASP) and illustrate its execution through the legal reasoning example described above. Section 4 shows the correctness of the implementation and its scalability is evaluated in Section 5. In Section 6 we discuss related work and Section 7 concludes the paper.

\section{Formalisation}

In this section we give the formal definition of our computational model. We first define the formal representation of past cases and related judgements. Using this representation, we define the concepts of relevant attack and prediction of judgements, and formalise the type of legal rules our approach is able to compute.

Definition 1 (Casebase). Let $F$ be a set of elements called factors. A case is a subset of $F$. A case with judgement is a pair $c j=\langle c, j\rangle$, where $c$ is a case and $j \in\{+,-\}$. The set $c$ is also referred to as the set of factors included in a case with judgement. Given a case with judgement $c j$, case $(c j)$ denotes the set of factors included in $\mathrm{cj}$ and judgement $(\mathrm{cj})=j$ denotes the judgement decision taken in the case. A casebase, denoted with $C B$, is a set of cases with judgements, namely a subset of $\mathcal{P}(F) \times\{+,-\}$, where $\mathcal{P}(F)$ is the powerset of $F$. 
Given a casebase $C B$ we impose the restriction that for every $c j_{1}, c j_{2} \in C B$, if $\operatorname{case}\left(c j_{1}\right)=\operatorname{case}\left(c j_{2}\right)$ then judgement $\left(c j_{1}\right)=j u d g e m e n t\left(c j_{2}\right)$. This avoids inconsistent casebases. We also assume that all casebases contain an element $\left\langle\emptyset, j_{0}\right\rangle$ representing the empty case and default judgement, the assumed judgement in the absence of any factor, of the casebase.

Definition 2 (Raw Attack). Let $C B$ be a casebase. The raw attack relation is a set $R A \subseteq C B \times C B$ defined as the set of all pairs $\left\langle c j_{1}, c j_{2}\right\rangle$ such that $\left\langle c j_{1}, c j_{2}\right\rangle \in R A$ if and only if case $\left(c j_{1}\right) \supset \operatorname{case}\left(c j_{2}\right)$ and judgement $\left(c j_{1}\right) \neq$ judgement $\left(c j_{2}\right)$. For every pair $\left\langle c j_{1}, c j_{2}\right\rangle \in R A$, we say $c j_{1}$ raw attacks $c j_{2}$ and we write $c j_{1} \rightarrow_{r} c j_{2}$.

Example 1. Let us consider the set of factors $F$ given by $\{a, b, c, d, e, f\}$ and a casebase $C B$ given by the following named cases with judgements:

$$
\left\{\begin{array}{lll}
c 0:\langle\{\},-\rangle & c 1:\langle\{a\},+\rangle & c 2:\langle\{c\},+\rangle \\
c 3:\langle\{a, b\},-\rangle & c 4:\langle\{a, b, c\},+\rangle & c 5:\langle\{a, b, c, d\},-\rangle
\end{array}\right\}
$$

Then, the raw attack relation over $C B$ is given by the following set:

$$
\left\{c 1 \rightarrow_{r} c 0, c 2 \rightarrow_{r} c 0, c 4 \rightarrow_{r} c 0, c 3 \rightarrow_{r} c 1, c 5 \rightarrow_{r} c 1, c 5 \rightarrow_{r} c 2, c 4 \rightarrow_{r} c 3, c 5 \rightarrow_{r} c 4\right\} .
$$

Definition 3 (Relevant Attack). Let $C B$ be a casebase. The relevant attack relation $A T \subseteq R A$ is the set of pairs $\left\langle c j_{1}, c j_{2}\right\rangle \in R A$ such that:

(i) $\left\langle c j_{1}, c j_{2}\right\rangle \in A T$ if $\operatorname{case}\left(c j_{2}\right)=\emptyset$ and there is no $c j_{3} \rightarrow_{r} c j_{2}$ in $R A$ such that $\operatorname{case}\left(c j_{1}\right) \supset \operatorname{case}\left(c j_{3}\right)$

(ii) $\left\langle c j_{1}, c j_{2}\right\rangle \in A T$ if there exists $\left\langle c j_{2}, c j_{4}\right\rangle \in A T$ and there is no $c j_{5} \rightarrow_{r} c j_{2}$ in $R A$ such that $\operatorname{case}\left(c j_{1}\right) \supset \operatorname{case}\left(c j_{5}\right)$

(iii) nothing else is in $A T$.

Each element $\left\langle c j_{1}, c j_{2}\right\rangle \in A T$ is denoted $c j_{1} \rightarrow c j_{2}$.

A relevant attack $c j_{1} \rightarrow c j_{2}$ is between a case $c j_{1}$ that overturns the judgement of another case $c j_{2}$, with $\operatorname{case}\left(c j_{1}\right) \supset \operatorname{case}\left(c j_{2}\right)$, and such that either judgement $\left(c j_{2}\right)=j_{0}$ or $c j_{2}$ itself is an attacker in another relevant attack; and there isn't another smaller attack against $c j_{2}$. Both scenarios imply that case $\left(c j_{1}\right)$ contains the relevant factors for overturning the judgement of $c j_{2}$. In summary, for a case to be relevant it must either be the default case, or it must be involved in a relevant attack against other relevant cases.

It can be observed from Definitions 2 and 3 that each casebase will have a unique set of raw attacks, and consequently a unique set of relevant attacks.

Example 2. Let us consider the set $R A$ of raw attacks defined in Example 1, The set of relevant attacks is given by the following subset:

$$
\{c 1 \rightarrow c 0, c 2 \rightarrow c 0, c 3 \rightarrow c 1, c 4 \rightarrow c 3, c 5 \rightarrow c 4, c 5 \rightarrow c 2\} .
$$

We can see that not all raw attacks are relevant attacks. Consider for instance the pair $\langle c 5, c 1\rangle$. This is a raw attack but it is not a relevant attack as there exists a raw attack $c 3 \rightarrow_{r} c 1$ where case $(c 3) \subset$ case $(c 5)$. For each relevant attack the set of factors, called argument, responsible for overturning the judgement can be deduced by comparing the factors in the two cases. 
Definition 4 (Argument). Let $C B$ be a casebase and let $A T$ be the set of relevant attack relation with respect to $C B$. For each pair $\left\langle c j_{1}, c j_{2}\right\rangle \in A T$, the set of factors $\alpha\left(c j_{1}, c j_{2}\right)$, representing the attack from $c j_{1}$ to $c j_{2}$, is given by $\alpha\left(c j_{1}, c j_{2}\right)=\operatorname{case}\left(c j_{1}\right)-\operatorname{case}\left(c j_{2}\right)$.

When predicting the judgement of a new case, we consider past cases similar to it. Within the set of similar past cases, only some will be active with respect to the new case, meaning their ruling has not been overturned by other cases in the set. Furthermore, those that are overturned cannot themselves overturn the judgement of other cases. This is similar to the concept of alive and dead node in [13]. We define active case with judgement as follows.

Definition 5 (Active Case with Judgement). Let $C B$ be a casebase, $A T$ be its corresponding set of relevant attacks, and $c$ be a case. A case with judgement $c j \in C B$ is active with respect to $c$ if and only if case $(c j) \subseteq c$, and for all $\left\langle c j_{n}, c j\right\rangle \in A T$, either case $\left(c j_{n}\right) \nsubseteq c$ or $c j_{n}$ is not active with respect to $c$.

We can infer the judgement of future cases from past cases' relevant attacks.

Definition 6 (Predicted judgement). Let $C B$ be a casebase, $A T$ be the set of relevant attacks with respect to $C B$, and $c$ be an unseen case (for all cj $\in C B$, case $(c j) \neq c)$. The unique predicted judgement of $c$, denoted with $p j(c)$, is equal to the default judgement $j_{0}$ if and only if $\left\langle\emptyset, j_{0}\right\rangle$ is active with respect to $c$.

The aim of this work is to generate a judgement theory $T$ from a given casebase $C B$ and default judgement $j_{0}$ such that, given a new case $c$ it is possible to predict the judgement of $c$.

\section{Generating Case-rules by ASP}

In this section we describe how we can reason about past cases to generate the case-rules, namely legal reasoning structures expressed in terms of principle rules and exceptions. This is done by using Answer Set Programming (ASP) and the Clingo 3 ASP solver [4]. We begin by describing how relevant attacks can be inferred from examples of past cases with judgements. Using this we describe how case-rules can be generated in the form of a meta-level representation.

Our ASP computational framework uses a meta-level representation of the casebase and the judgement theory. Its reasoning process can be divided into three main steps: (i) extraction of relevant attacks from the given casebase; (ii) inference of the factors in the arguments of each relevant attack; iii) generation of the judgement theory using the arguments. These steps are shown in Figure 1 , whose labelled programs are explained further in this section.

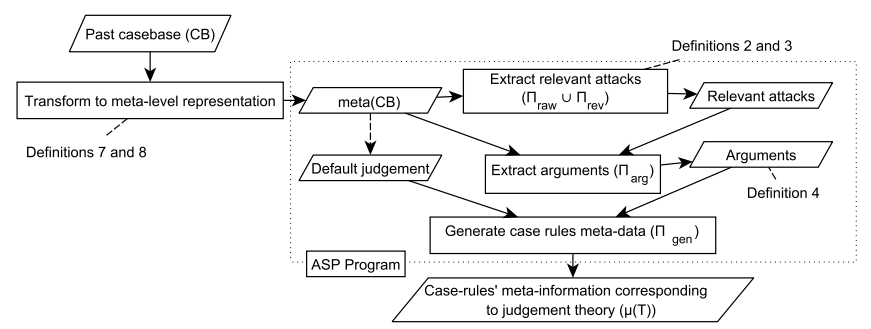

Fig. 1. Work flow for generating case-rules by ASP 


\subsection{Extracting Relevant Attacks from a Casebase}

Each casebase can be seen as a definite clause.

Definition 7 (Rule representation of a case with judgement). Let $C B$ be a casebase. Each $c j \in C B$ can be expressed as a definite clause $r(c j)$ :

$$
\text { judgement }(c j) \leftarrow f_{1} \wedge \ldots \wedge f_{n} \text {. }
$$

where $f_{i} \in$ case $(c j)$, for $1 \leq i \leq n$. We refer to such a clause as a rule.

Inferring relevant attacks and arguments means reasoning about the structure of the set of rules that express a given casebase. The meta-level representation of our cases with judgement describes the syntactic structure of each case in terms of the literals (i.e. factors) that appear in each case.

Definition 8 (Casebase meta-level representation). Let $C B$ be a casebase. Its meta-level representation, meta $(C B)$, is defined as:

$$
\operatorname{meta}(C B)=\bigcup_{c j \in C B} \mu(r(c j)) \cup \tau(C B) \cup \delta(C B)
$$

$\tau(C B)=\left\{\right.$ factor $\left.\left(\mathrm{f}_{\mathrm{i}}\right) \mid \mathrm{f}_{\mathrm{i}} \in \mathrm{F}\right\}, \delta(C B)$ is the meta-information about the default case, $\delta(C B)=\left\{\right.$ default_id $\left(\mathrm{id}\left(\mathrm{r}\left(\mathrm{c} \mathrm{j}_{0}\right)\right)\right)$, default_head $\left.\left(\mathrm{judgement}_{(\mathrm{cj} 0)}\right)\right\}$, and the function $\mu$ is defined as follows:

$$
\mu(r(c j))=\left\{\begin{array}{l}
\text { cb_id }(i d(r(c j))) . \\
i s_{2} r u l e(i d(r(c j)), j u d g e m e n t(c j)) . \\
i n_{\_} r u l e\left(i d(r(c j)), j u d g e m e n t(c j), f_{i}\right) . \quad \text { for each } f_{i} \in \operatorname{case}(c j)
\end{array}\right.
$$

This meta-level representation can be used to express the notion of raw attack $\left(\rightarrow_{r}\right)$ given in Definition 2. The second and third rules below capture the condition $\operatorname{case}\left(c j_{1}\right) \supset \operatorname{case}\left(c j_{2}\right)$, where $I D_{1}$ and $I D_{2}$ are the IDs of cases with judgement $c j_{1}$ and $c j_{2}$ respectively; the condition $H_{1} \neq H_{2}$ in the first rule below captures the condition judgement $\left(c j_{1}\right) \neq j$ judgement $\left(c j_{2}\right)$.

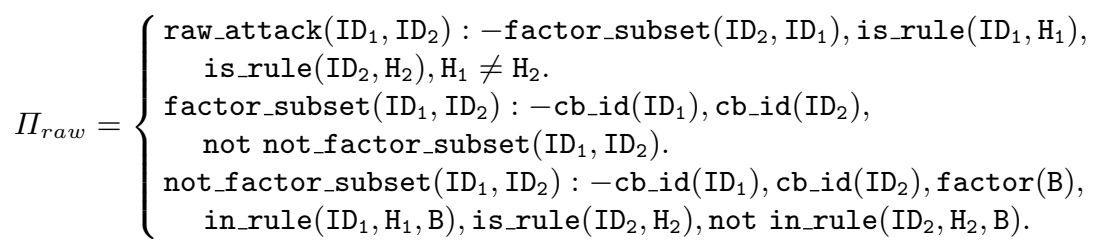

The computation of the relevant attack relation (called attack in the program) uses ASP's choice operator (see first rule below) to select from the inferred raw attacks those relations that satisfy constraints (i) and (ii) given in Definition 3. They are captured by the second and third rules given below.

$$
\Pi_{\text {rev }}=\left\{\begin{array}{l}
0\left\{\operatorname{attack}\left(\mathrm{ID}_{1}, \mathrm{ID}_{2}\right)\right\} 1:- \text { raw_attack }\left(\mathrm{ID}_{1}, \mathrm{ID}_{2}\right) . \\
:-\operatorname{attack}\left(\mathrm{ID}_{1}, \mathrm{ID}_{2}\right), \text { not attackee }\left(\mathrm{ID}_{2}\right) . \\
:-\operatorname{attack}\left(\mathrm{ID}_{1}, \mathrm{ID}_{2}\right), \text { raw_attack }\left(\mathrm{ID}_{3}, \mathrm{ID}_{2}\right), \text { factor_subset }\left(\mathrm{ID}_{3}, \mathrm{ID}_{1}\right) . \\
\text { attackee }(\mathrm{ID}):-\operatorname{def} \text { ault_id }(\mathrm{ID}) . \\
\text { attackee }\left(\mathrm{ID}_{2}\right):-\operatorname{attack}\left(\mathrm{ID}_{2}, \mathrm{ID}_{4}\right) .
\end{array}\right.
$$

To generate all relevant attacks in a given casebase, we use the following Clingo optimisation expression, which guarantees the maximum number of instances of the attack relation to be computed in a given solution. 


$$
\Pi_{\text {rev }_{2}}=\left\{\# \text { maximise }\left\{\operatorname{attack}\left(\mathrm{ID}_{1}, \mathrm{ID}_{2}\right)\right\} .\right\}
$$

Let $\Pi_{r e v}=\Pi_{r e v_{1}} \cup \Pi_{r e v_{2}}$, and let $\Pi_{C B}=\operatorname{meta}(C B) \cup \Pi_{\text {raw }} \cup \Pi_{r e v}$. The answer set of meta $(C B) \cup \Pi_{\text {raw }}$ gives the subfactors and raw attacks between cases in the casebase. This is then used by $\Pi_{\text {rev }}$ to generate all the relevant attacks $A T$. Thus the answer set of $\Pi_{C B}$ contains the meta-level representation of the casebase, subfactors, raw attacks, relevant attacks and attackees.

\subsection{Generating Meta-level Information of Case-rules}

Using inferred relevant attacks and the meta-level representation of given cases, we can compute the set of arguments $A R$ of the relevant attacks:

$\Pi_{\text {arg }}=\left\{\begin{array}{r}\operatorname{argument}\left(\mathrm{ID}_{1}, \mathrm{ID}_{2}, \mathrm{Arg}\right):-\operatorname{attack}\left(\mathrm{ID}_{1}, \mathrm{ID}_{2}\right), \text { in_rule }\left(\mathrm{ID}_{1}, \mathrm{H}_{1}, \mathrm{Arg}\right), \\ \text { is_rule }\left(\mathrm{ID}_{2}, \mathrm{H}_{2}\right), \text { not in_rule }\left(\mathrm{ID}_{2}, \mathrm{H}_{2}, \mathrm{Arg}\right) .\end{array}\right.$

In order to predict the judgement of unseen cases, our judgement theory reflects the underlying reasoning applied throughout the past judged cases. The legal reasoning structure is normally composed of exceptions to a given default assumption (e.g. default judgement) and exceptions to exceptions.

Definition 9. Given a casebase $C B$ and its relevant attacks $A T$, the judgement theory $T$ is the set of rules such that given a new case $c, T$ derives the default judgement $j_{0}$ if and only if $p j(c)=j_{0}$. Let $a b\left(c j_{i} \rightarrow c j_{j}\right)$ be the reified atom of $c j_{i} \rightarrow c j_{j}$ in AT. The following rules are in the judgement theory:

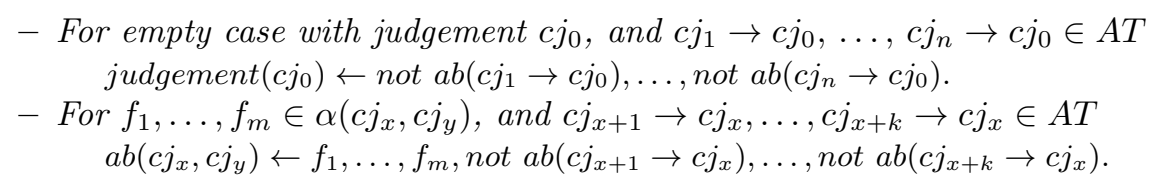

Our computational approach abduces the above judgement theory in terms of its equivalent meta-level representation $\mu(T)$. The judgement theory itself is acquired by applying the inverse transformation $\mu^{-1} / 1$. For example, $\mu^{-1}$ applied to the set of instances $\left\{i s_{-} r u l e(i d, h)\right.$. in_rule $\left(i d, h, b_{1}\right) . \quad \ldots \quad$ in_rule $\left.\left(i d, h, b_{n}\right).\right\}$ gives the rule $h \leftarrow b_{1}, \ldots, b_{n}$.

To generate a legal reasoning structure each relevant attack inferred from the past cases has to be linked to a unique abnormality name. This Skolemisation is captured using the ASP choice rule given below, which associates the attack identifier $A I D$ of a given attack $a\left(I D_{1}, I D_{2}\right)$ with an abnormality name $A b$. An integrity constraint guarantees that attack identifiers are unique.

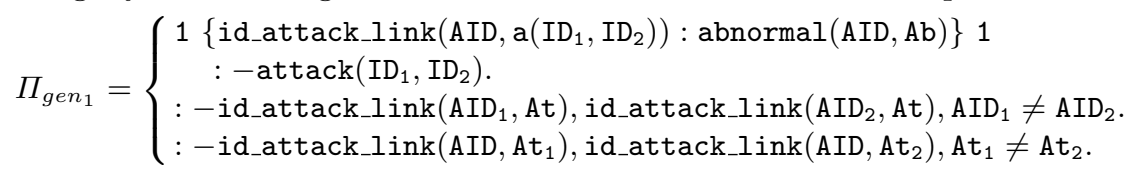

Abnormality names are defined by the basic types abnormal and negated_abnormal as illustrated below:

$\Pi_{g^{\prime} n_{2}}=\left\{\begin{array}{l}\operatorname{gen} \_i d(\mathrm{r} 0) . \\ \operatorname{gen} \_\mathrm{id}(\mathrm{ri}) . \text { abnormal}(\mathrm{ri}, \mathrm{abi}) . \text { negated_abnormal(ri,not_abi). } \\ \quad \text { For } 1 \leq i \leq n, \text { where } n \text { is the number of relevant attacks }\end{array}\right.$

Once appropriate links between relevant attacks and abnormal identifiers are obtained through the choice rule given above, the meta-level representation of 
the rules defining these abnormalities can be inferred using the following two sets of rules. The first rule expresses the fact that the default judgement is the judgement of an empty factor set, thus it can only be applied on future cases if all attacks against cannot be proved to hold. The second rule captures the metalevel representation of an principle rule of the form $j_{0} \leftarrow$ not $a b_{1}, \ldots$, not $a b_{m}$, defining the absence of exceptions to the default judgement.

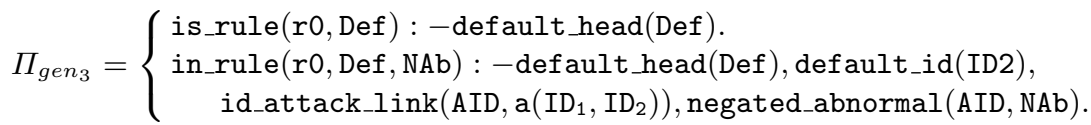

For each abduced link between relevant attack and abnormality names, the following set of rules allows the inference of the meta-level representation of rules of the form $a b_{i} \leftarrow f_{i 1}, \ldots, f_{i k_{i}}$, not $a b_{m i 1}, \ldots$, not $a b_{p}$ and $a b_{q} \leftarrow f_{q 1}, \ldots, f_{q k_{q}}$.

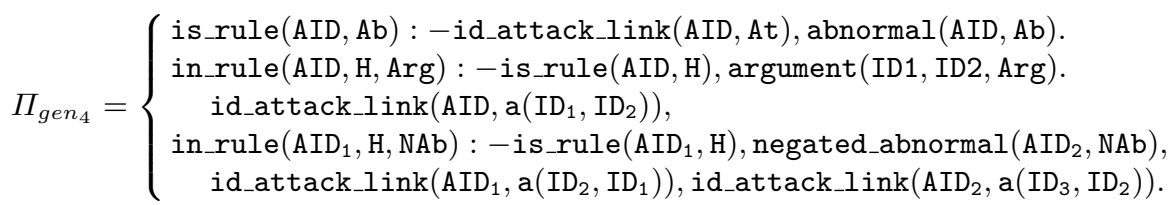

The first two rules allow the inference of the definition of predicate head abnormality name $A b$ that corresponds to the correct linked attack, for which the appropriate factors involved in the argument of this attack are inferred to be conditions in the body of such abnormality rule. The third rule captures the abnormality identifiers of subsequent attacks that invalidate the current exception.

Let $\Pi_{g e n}=\Pi_{g e n_{1}} \cup \Pi_{g e n_{2}} \cup \Pi_{g e n_{3}} \cup \Pi_{g e n_{4}}$, and let $\Pi_{J T}=\Pi_{g e n} \cup A R \cup$ $\left\{\right.$ default_head $\left(j u d g e m e n t\left(c j_{0}\right)\right)$, default_id $\left.\left(i d\left(r\left(c j_{0}\right)\right)\right)\right\}$. The answer set of the program $\Pi_{J T}$ projected over $i s_{-}$rule $/ 2$ and in_rule/2 corresponds to the metalevel representation of the judgement theory $\mu(T)$.

\subsection{Application to Legal Reasoning Example}

We show the result of our approach applied to the past cases with judgements described in Section $1^{3}$. The following meta-level representation of the judgement theory is generated from $\Pi_{g e n}$ :

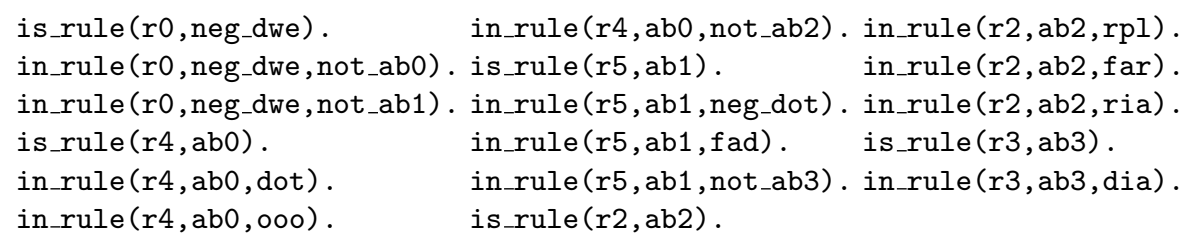

This corresponds to the program:

neg_dwe :- not ab0, not ab1. ab2 :- far, ria, rpl.

ab0 :- dot, ooo, not ab2. ab3 :- dia. ab1 :- fad, neg_dot, not ab3.

\section{Correctness of the Generated Program}

We prove the correctness of the program through two propositions. Proposition 1 ensures that all relevant attacks of the given casebase are computed,

\footnotetext{
3 The full example with each ASP program's output and proofs for the lemmas can be found at www.doc.ic.ac.uk/ da407/lpnmr2015/supplementary.pdf
} 
while Proposition 2 ensures the correct predicted judgement is computed. The proof of Proposition 1 is divided into four steps ${ }^{3}$. In Lemma 1, we establish the properties of an answer set of $\Pi_{C B}$. Lemma 2 shows that the union of two answer sets of $\Pi_{C B}$ is also an answer set of $\Pi_{C B}$. In Lemma 3 , we show that ans $(C B, A T) \in A S\left(\Pi_{C B}\right)$, where $A S(P)$ is the set of answer sets of a given program $P$, and ans $(C B, A T)$ is defined below.

Definition 10. Given a casebase $C B$ with relevant attacks $A T$, ans $(C B, A T)$ is the program $S \cup Q \cup\left\{\operatorname{attackee}\left(i d\left(r\left(c j_{0}\right)\right)\right)\right\}$, where $\{S\}=A S\left(\operatorname{meta}(C B) \cup \Pi_{\text {raw }}\right)$ and $Q=\left\{\operatorname{attack}\left(i d\left(r\left(c j_{i}\right)\right), i d\left(r\left(c j_{j}\right)\right)\right)\right.$, attackee $\left.\left(i d\left(r\left(c j_{j}\right)\right)\right) \mid\left\langle c j_{i}, c j_{j}\right\rangle \in A T\right\}$.

These lemmas are used to show that ans $(C B, A T)$ is the unique optimal answer set of $\Pi_{C B}$.

Lemma 1. Let $S$ be the unique answer set of meta $(C B) \cup \Pi_{\text {raw }}$ and let $\pi_{p}(A)$ denote the projection of $A$ over $p$. A set $A \in A S\left(\Pi_{C B}\right)$ iff:

$i \pi_{\text {attack }}(A) \subseteq \pi_{\text {raw_attack }}(A)$

ii For all $c j_{1}$ and $c j_{2}$, if attack $\left(i d\left(r\left(c j_{1}\right)\right), i d\left(r\left(c j_{2}\right)\right)\right) \in A$ then attackee $\left(i d\left(r\left(c j_{2}\right)\right)\right) \in A$

iii For all $c j_{1}$ and $c j_{2}$, if attack $\left(i d\left(r\left(c j_{1}\right)\right), i d\left(r\left(c j_{2}\right)\right)\right) \in A$ then there does not exists $\quad c j_{3}$ such that raw_attack $\left(i d\left(r\left(c j_{3}\right)\right), i d\left(r\left(c j_{2}\right)\right)\right) \in A$ and factor_subset $\left.\left(i d\left(r\left(c j_{3}\right)\right), i d\left(r\left(c j_{1}\right)\right)\right) \in A\right)$

iv For all $c j$, attackee $\left(i d(r(c j)) \in A\right.$ if and only if default_id(id $\left(r\left(c j_{3}\right)\right)$ is true or attack $\left(i d(r(c j)), i d\left(r\left(c j_{x}\right)\right)\right) \in A$

$v$ Let $\mathcal{L}$ be the language of meta $(C B) \cup \Pi_{\text {raw }}$, then for all $s, s \in \pi_{\mathcal{L}}(A)$ if and only if $s \in S$

Lemma 2. Given two answer sets $A_{1}, A_{2} \in A S\left(\Pi_{C B}\right)$, then $A_{1} \cup A_{2} \in A S\left(\Pi_{C B}\right)$.

Lemma 3. Given a casebase $C B$, its raw attacks $R A$, and its relevant attack $A T, \operatorname{ans}(C B, A T) \in A S\left(\Pi_{C B}\right)$.

Proposition 1. Given a casebase $C B$ with relevant attacks $A T$, ans $(C B, A T)$ is the unique optimal answer set of $\Pi_{C B}$.

Proof. By Lemma 3, ans $(C B, A T) \in A S\left(\Pi_{C B}\right)$. Assume, by contradiction, that there exists $A_{\text {opt }}^{\prime} \in A S\left(\Pi_{C B}\right)$ such that $A_{\text {opt }}^{\prime} \neq$ ans $(C B, A T)$ and $A_{\text {opt }}^{\prime}$ is more or equally optimal to ans $(C B, A T)$. As both $A_{\text {opt }}^{\prime}$ and ans $(C B, A T)$ satisfy (v) and attackee/ 1 is defined using attack/2 then there must be an attack instance in $A_{\text {opt }}^{\prime}$ which is not in ans $(C B, A T)$. Let $D=A_{o p t}^{\prime} \backslash$ ans $(C B, A T)$. By Lemma 2 $A_{\text {opt }}^{\prime} \cup$ ans $(C B, A T)$ must also be an answer set of $\Pi_{C B}$, furthermore $A_{\text {opt }}^{\prime} \cup$ ans $(C B, A T)=D \cup$ ans $(C B, A T)$. Let $d$ be an attack/2 instance such that $\operatorname{attack}\left(i d_{1}, i d_{2}\right) \in D$ and for all $i d_{3}$ such that $\operatorname{attack}\left(i d_{2}, i d_{3}\right) \notin D$. Let $A^{\prime}=$ $\{d\} \cup\left\{\operatorname{attackee}\left(i d_{1}\right)\right\} \cup \operatorname{ans}(C B, A T)$. $A^{\prime}$ satisfies (i), (iii), and (v) as they are also satisfied by $D \cup$ ans $(C B, A T)$. As $d \in A_{\text {opt }}^{\prime}$ then either default $\left(i d_{2}\right) \in A_{\text {opt }}^{\prime}$ or $\operatorname{attack}\left(i d_{2}, i d_{3}\right) \in A_{\text {opt }}^{\prime}$. In the first case default $\left(i d_{2}\right) \in \operatorname{ans}(C B, A T \cup\{d\})$ and in the second since $\operatorname{attack}\left(i d_{2}, i d_{3}\right) \in A_{o p t}^{\prime}$ but $\operatorname{attack}\left(i d_{2}, i d_{3}\right) \notin D$ it must be the case that $\operatorname{attack}\left(i d_{2}, i d_{3}\right) \in A_{\text {opt }}^{\prime} \cap \operatorname{ans}(C B, A T)$ and hence $\operatorname{attack}\left(i d_{2}, i d_{3}\right) \in A^{\prime}$. So in both cases $A^{\prime}$ satisfies (ii). Lastly since ans $(C B, A T)$ satisfies (iv), so would $A^{\prime}$. This shows that $A^{\prime}$ is also an answer set of $\Pi_{C B}$. However, let $\left\langle c j_{1}, c j_{2}\right\rangle$ be 
the relevant attack corresponding to $d$, then by (ii), (iii) and (iv) and Definition 3 then $\left\langle c j_{1}, c j_{2}\right\rangle \in A T$ must be true and so $d \in$ ans $(C B, A T)$. Contradiction as $d \in A_{\text {opt }}^{\prime} \backslash$ ans $(C B, A T)$. Thus ans $(C B, A T)$ is the unique optimal answer set of $\Pi_{C B}$.

We assume that $\Pi_{J T}$ will generate the correct judgement theory as described by Definition 9 . The proof of Proposition 2 is divided into two parts. Lemma 6 shows that the judgement theory can be partitioned into one part $T_{c}$ responsible, and another $T_{e x}$ irrelevant for the derivation of $j_{0}$. We then show that $T_{c}$ derives the $j_{0}$ if and only if it is the predicted judgement.

Lemma 4. Given a casebase $C B$ and associated judgement theory $T$. Then for $A \in A S\left(\Pi_{J T}\right), \pi_{\text {in_rule,is_rule }}(A)=\mu(T)$.

For rule $r \in T$, let head $(r)$ be its head literal, $\operatorname{body}(r)$ be the set of its body literals, $f s(r)$ be the set of factors in its body literal, and def be a rule where head $($ def $)=j_{0}$. The following property can be derived from Definition 9 .

Lemma 5. Given a casebase $C B$ with associated judgement theory $T$, let $c$ be a new case, given as a set of factors. From Definition 9 for all abnormality rules $a b$ in $T$ there exists a sequence of rules not head $(a b) \in \operatorname{body}\left(a b_{x_{1}}\right), \ldots$, not head $\left(a b_{x_{n}}\right) \in \operatorname{body}(\operatorname{def})$ in $T$, where $n \geq 0$. The union of all its factors corresponds to a $c j \in C B$, and $c j \rightarrow c j_{y}$ for some $c j_{y} \in C B$. Abnormalities with a sequence such that case $(c j) \subseteq c$ is denoted by seq $(a b)$.

Lemma 6. Given a casebase $C B$ with associated judgement theory $T$, and a new case c. Let $T_{c}=\{d e f\} \cup\{a b \mid a b \in T$, seq $(a b)\}$, and $T_{e x}=T \backslash T_{c}$. Then $T$ derives $j_{0}$ iff $T_{c}$ derives $j_{0}$

Proposition 2. Given a casebase $C B$ with associated judgement theory $T$, a new case $c$, let and $\left\{A_{T}\right\}=A S(T \cup c)$. Then $j_{0} \in A_{T}$ if and only if $p j(c)=j_{0}$.

Proof. Let $A_{c}$ be the answer set of $T_{c}$, and let $C B_{c}$ be the set of $c j \in C B$ such that case $(c j) \subseteq c$, and judgement $(c j)=j_{0}$ or $c j \rightarrow c j_{y}$ for some $c j_{y} \in C B$. By Lemma 6 we can reduce the problem to $j_{0} \in A_{c}$ if and only if $p j(c)=j_{0}$. This can be shown inductively for each $r \in T_{c}$ :

- Base case: $r$ corresponding to $c j \in C B_{c}$ with largest case is satisfiable. There does not exist $c j^{\prime} \in C B_{p j}$ where $\operatorname{case}(c j) \subset c a s e\left(c j^{\prime}\right)$, thus $c j$ must be active. All $f \in f s(a b)$ must be in $c$, and all not $a b \in \operatorname{body}(r)$ hold as they must be in $T_{e x}$. Thus head $(r) \in A_{c}$.

- Inductive hypothesis: Let $r$ has corresponding $c j \in C B_{c}$. For all $r^{\prime}$ corresponding to $c j^{\prime} \in C B_{c}$ such that $\operatorname{case}(c j) \subset \operatorname{case}\left(c j^{\prime}\right), r^{\prime}$ is satisfiable if and only if $c j^{\prime}$ is an active case with respect to $c$.

- Inductive step: $r$ corresponding to $c j \in C B_{c}$ with case that is not the largest in $C B_{c}$. All $f \in f s(r)$ are in $c$, and for each not $a b \in \operatorname{body}(r), a b$ corresponds to $c j^{\prime} \in C B$ such that $\operatorname{case}(c j) \subset \operatorname{case}\left(c j^{\prime}\right)$. By the inductive hypothesis each $a b$ is satisfiable iff $c j^{\prime}$ is active for $c$. So by Definition $5, c j$ is active if and only if all not $a b$ are satisfiable. Thus, head $(r) \in A_{c}$ if and only if $c j$ is active with respect to $c$. 


\section{Evaluation}

To test the performance of the approach we have applied it to randomly generated (consistent) casebases where the number of cases ranges from 20 to 100 , and the number of factors ranges from 10 to 25 . The time taken to generate the meta-level representation of the judgement theory for each casebase is presented in Figure 2. To mitigate the grounding problem, the ASP program is split into two parts with the first program $\Pi_{C B} \cup \Pi_{\text {arg }}$ used for generating the relevant attacks and arguments (Figure 2 (a)), which are then added as facts to the second program $\Pi_{g e n}$ to generate the judgement theory (Figure 2 (b)). Both Figures 2 (a) and (b) show that the computational time increases with the number of cases, while the change in the number of factors is less important. It can be seen that the times in Figure 2 (a) are not significant when compared with those in Figure 2 (b). The reason for the much larger times in Figure 2 (b) is due to the use of Skolemisation in $\Pi_{g e n}{ }^{4}$. However, the computational time of $\Pi$ could be greatly decreased by using an external process to perform the Skolemisation, which would allows the generation of the judgement theory for casebases with greater than 100 cases.
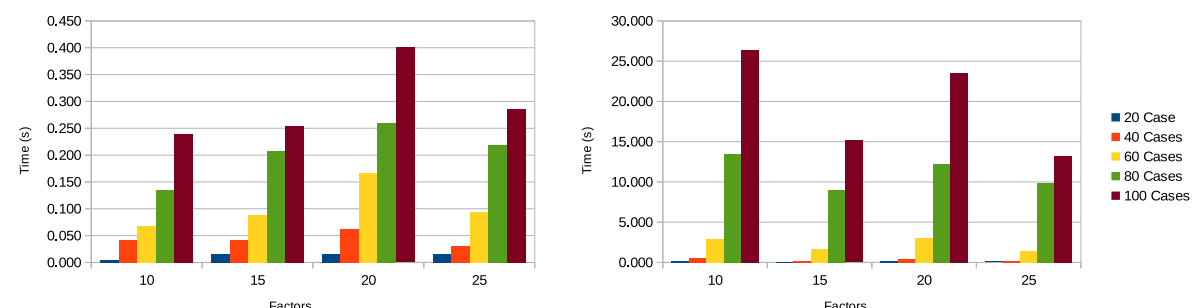

Fig. 2. Computational time for (a) $\Pi_{C B} \cup \Pi_{\text {arg }}$ to output $A_{\text {atk,arg }}$; (b) $\Pi_{\text {gen }} \cup$ $\pi_{\text {attack,argument,default_id,default_head }}\left(A_{a t k, a r g}\right)$ applied to randomly generated $C B$

\section{Related Work}

In this work we have shown how meta-level reasoning can be used for extracting information from past legal cases for generating case rules for deciding the judgement of future cases. We have used notions of argumentation and ASP for computation, and while there have been many recent works [14] in representing argumentation framework and computing argumentation extensions using ASP, we are concerned with the extraction of information about the arguments and attacks from examples rather than computing extensions of a given framework.

Similar to legislators using past legal cases for creating or revising legislations, past work in legal reasoning has explored how formal reasoning can be applied for reasoning from past cases $[9,10]$, or [11] where a boolean function is used for casebase classification and reasoning. The system HYPO [1] also analyses factors in the form of dimensions, where a dimension is a structure containing a factor and the party it favours, to suggest the arguments and counter examples that

\footnotetext{
${ }^{4}$ While we were able to compute the relevant attacks and arguments for casebase with greater than 100 cases, we were unable to generate the judgement theory from them using $\Pi_{g e n}$.
} 
plaintiff and defendant may use to further their objectives. It sorts case relevance using a claim lattice, a directed acyclic graph of dimensions and cases relevant to the set of dimensions. This differs from our approach where we sort cases using the relevant attacks, arranging them according to their ability to overturn judgements of other cases. Additionally, [8] shows how meta-level representation can be used for legal reasoning, however this concerns the representation and interpretation of the rules, and not the generation of rules.

The legal reasoning system PROLEG is used to represent rules and exceptions [12]. In fact, there is a correspondence between the representation used in PROLEG and the rules that we generate. PROLEG rules do not use negation as failure explicitly; exceptions are expressed by the form exception $(H, E)$ where $H$ and $E$ are atoms. The generated rule $C:-B_{1}, \ldots, B_{n}, \operatorname{not} E_{1}, \ldots, \operatorname{not} E_{m}$, where $n \geq 0, m \geq 0$, is represented in PROLEG as

$$
C \Leftarrow B_{1}, \ldots, B_{n} . \quad \operatorname{exception}\left(C, E_{1}\right) . \quad \ldots \quad \operatorname{exception}\left(C, E_{m}\right) .
$$

For instance, the judgement theory we generated in in Section 3.3 can be translated into the following PROLEG program.

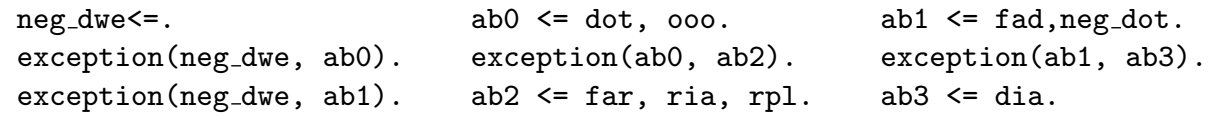

Therefore, our approach can also be regarded as generating PROLEG programs.

Inductive learning has often been used for learning such rules, using multiple learning phases to learn exceptions. For instance in [6] and [7], the learning is split into two phases, the first phase learns the overly general rules from the examples, and the second phase specialises the general rules using exceptions. Our work is similar to the second phase, but with an assumed over-general rule (default judgement) to be given from the legal specification. Other similar work (e.g. [2]) uses prioritised logic programming to express preferences between the default rules.

\section{Conclusion}

We have presented a method for reasoning and extracting information from past cases to infer the arguments and attacks present in the decision for the judgement of a new case. We have defined the notion of minimal attacks for identifying the factors relevant to the judgements of cases, and describe how ASP can be used to generate these minimal attacks as well as how these attacks can be used for inferring rules for modelling the judgement using meta-level information. While not shown in this paper, PROLOG could be used instead of ASP, using its list structure to represent the rules.

For future work, it would be interesting to expand the approach to consider dependencies between factors, by extending the meta-level representation and enhancing the reasoning approach for handling more complex casebases. For instance, in [5], a different representation of the casebase is used where information on whether a factor favours the defendant or plaintiff. This allows for factors to be grouped together according to side it favours, and for reasoning to be applied on groups of like sided factors. 
Acknowledgment. This work was partially supported by JSPS KAKENHI Grant Numbers 26280091. The authors would like to thank Robert Kowalski for his valuable comments, and Kristijonas Cyras for his two examples, including Example 1, which indicated an error in the paper presented at the Eighth International Workshop on Juris-informatics (JURISIN 2014), Kanagawa, Japan.

\section{References}

1. Kevin D. Ashley. Reasoning with cases and hypotheticals in hypo. In: Int. J. ManMachine Studies, 34:753-796, 1991.

2. Yannis Dimopoulos and Antonis Kakas. Learning non-monotonic logic programs: Learning exceptions. In Machine Learning: ECML-95, volume 912 of LNCS, pages 122-137. 1995.

3. Phan Minh Dung. On the acceptability of arguments and its fundamental role in nonmonotonic reasoning, logic programming and n-person games. Artificial Intelligence, 77:321-357, 1995.

4. Martin Gebser, Benjamin Kaufmann, Roland Kaminski, Max Ostrowski, Torsten Schaub, and Marius Thomas Schneider. Potassco: The potsdam answer set solving collection. AI Commun., 24(2):107-124, 2011.

5. John F. Horty. Reasons and precedent. In Kevin D. Ashley and Tom M. van Engers, editors, The 13th International Conference on Artificial Intelligence and Law, Proceedings of the Conference, Pittsburgh, USA, pages 41-50. ACM, 2011.

6. Katsumi Inoue. Learning extended logic programs. In In Proceedings of the 15th International Joint Conference on Artificial Intelligence, pages 176-181, 1997.

7. Kouzou Ohara, Hideyuki Taka, Noboru Babaguchi, and Tadahiro Kitahashi. Determination of general concept in learning default rules. In PRICAI 2000 Topics in Artificial Intelligence, volume 1886 of LNCS, pages 104-114. 2000.

8. Tom Routen and Trevor J. M. Bench-Capon. Hierarchical formalizations. International Journal of Man-Machine Studies, 35(1):69-93, 1991.

9. Ken Satoh. Translating case-based reasoning into abductive logic programming. In 12th European Conference on Artificial Intelligence, Budapest, Hungary, 1996, Proceedings, pages 142-146, 1996.

10. Ken Satoh. Statutory interpretation by case-based reasoning through abductive logic programming. JACIII, 1(2):94-103, 1997.

11. Ken Satoh. Analysis of case-based representability of boolean functions by monotone theory. In Algorithmic Learning Theory, 9th International Conference, ALT '98, Germany, 1998, Proceedings, volume 1501 of LNCS, pages 179-190, 1998.

12. Ken Satoh, Kento Asai, Takamune Kogawa, Masahiro Kubota, Megumi Nakamura, Yoshiaki Nishigai, Kei Shirakawa, and Chiaki Takano. PROLEG: an implementation of the presupposed ultimate fact theory of japanese civil code by PROLOG technology. In New Frontiers in Artificial Intelligence - JSAI-isAI 2010 Workshops, Tokyo, Japan, 2010, volume 6797 of LNCS, pages 153-164, 2010.

13. Ken Satoh, Masahiro Kubota, Yoshiaki Nishigai, and Chiaki Takano. Translating the japanese presupposed ultimate fact theory into logic programming. In JURIX 2009: The Twenty-Second Annual Conference on Legal Knowledge and Information Systems, The Netherlands, 2009, pages 162-171. IOS Press, 2009.

14. Francesca Toni and Marek Sergot. Argumentation and answer set programming. In Logic Programming, Knowledge Representation, and Nonmonotonic Reasoning, volume 6565 of LNCS, pages 164-180. 2011. 